\title{
Balancing of flexible rotors based on evolutionary algorithms
}

\author{
Wasiu Adeyemi Oke ${ }^{1, a}$, Mohammad Ali Abido ${ }^{2}$ and Tesleem Babatunde Asafa ${ }^{1}$ \\ 1 Mechanical Engineering Department, King Fahd University of Petroleum and Minerals, P.O Box 8611, Dhahran, Saudi Arabia \\ 2 Electrical Engineering Department, King Fahd University of Petroleum and Minerals, P.O Box 1225, Dhahran, Saudi Arabia
}

Received 1 January 2014, Accepted 3 November 2014

\begin{abstract}
An unbalance in a rotating flexible rotor causes excessive vibration and elastic deformations with subsequent malfunction and failure. In spite of different techniques deployed to reduce or eliminate rotor unbalance, it is impossible to remove the unbalance completely. The unbalance will only be reduced to a residual level. Hence, any other method that can reduce this residual level further can be considered as an alternative. In this article, Differential Evolution (DE) and Genetic Algorithm (GA) were successfully applied as optimization techniques to balance rotating flexible rotors. The unbalancing challenge is formulated as an optimization problem with an objective function of minimizing the rotor unbalance by identifying the optimum correction parameters. Modeling and response analyses were performed in ANSYS while optimizations were conducted in MATLAB. The results of four balancing cases show that the approaches are robust at both balancing speed and beyond. Also, the results obtained show that GA performs slightly better than DE in terms of optimization time and effective reduction of vibration amplitude.
\end{abstract}

Key words: Optimization / rotor unbalance / evolutionary algorithms / ANSYS / MATLAB

\section{Introduction}

Rotating machinery accounts for majority of presentday machines. While the dynamics of such machinery has been important, the need for higher efficiency has consequently led to design of machines which are increasingly liable to vibration at higher speeds. A level of unbalance in a rotor that is tolerable at a low speed might be intolerable at a higher speed because the centrifugal force due to an unbalance could be coupled with an elastic deformation of the rotor. An unbalance in a rotating system can have devastating effects during start-up, slow down or when operating at or near critical frequency. Such an unbalance may be due to material faults, manufacturing and assembly errors, transportation and commissioning as well as operational conditions. Consequently, noises, wears, high level of vibrations, reduction in machine life, etc, are expected. In flexible rotors (such as high-speed turbines, generators and paper-machine rolls), unbalance problem becomes more complicated due to the interaction with the elastic deformations of the rotor, and thus calls for a special balancing procedure.

Rotor balancing can be done either by adding or removing a correction mass of which its quantity and location can be quantified either by modal or influence coefficient method [1-7]. Modal balancing is a progressive or mode-by-mode balancing method [2]. The influence

${ }^{a}$ Corresponding author: wasiuad@kfupm.edu.sa coefficient method is a data processing technique aiming at minimizing rotor's vibration to an acceptable level and a relationship between the two approaches has been developed by Tan and Wang [3]. It was stated in reference [3] that for the influence coefficient approach, this relationship requires that the rotor-bearing systems should be undamped or have insignificant damping and the effect of run speeds on its natural frequencies should be negligible. However, the applicability of the method has not been demonstrated and it can be inferred that their proposed relationship between modal and influence coefficient methods is not applicable to all rotors because there are rotors with significant damping. Other methods employed for balancing rotors available in the literatures include: holo-balancing method by Liu and $\mathrm{Qu}[4]$ where two sensors were used at each measuring point and the method is limited to the rotors that are operating between the first and second critical speeds. Xu et al. [6] used transfer matrix and genetic algorithm to balance the rotor and two sensors were utilized at each measurement point. Active vibration control scheme was proposed by Das et al. [8] in which electromagnetic exciters were employed. Nevertheless, their approach cannot be applied to all rotors because the exciters will constraint the bearing or disk movement where the shaft is not long or disks are closed together. Other available method is the minimization of Hermitian matrix of influence coefficients by Kang et al. [9]. 


\section{Nomenclature}

\begin{tabular}{|lll|}
\hline Notation & Unit & Description \\
$\ddot{\boldsymbol{y}}$ & $\mathrm{N} \cdot \mathrm{m}^{-2}$ & Acceleration vector \\
$\boldsymbol{A} \boldsymbol{m}$ & $\mathrm{m}$ & Amplitude matrix \\
$Y_{i}$ & $\mathrm{~m}$ & Amplitude of $\boldsymbol{Y}_{i}$ \\
$C_{x x}, C_{y y}$ & $\mathrm{Ns} \cdot \mathrm{m}^{-1}$ & Bearings damping coefficients \\
$K_{x x}, K_{y y}$ & $\mathrm{~N} \cdot \mathrm{m}^{-1}$ & Bearings Stiffness coefficients \\
$\boldsymbol{C}$ & $\mathrm{Ns} \cdot \mathrm{m}^{-1}$ & Damping matrix for bearings and gyroscopic of the shaft and disks \\
$r$ & $\mathrm{~m}$ & Distance from unbalance mass to shaft/rotor centerline \\
$\boldsymbol{q}$ & $\mathrm{N}$ & Force vector \\
$i$ & & Generation number \\
$m_{l}$ & $\mathrm{~kg}$ & Lower boundary of trial correction mass \\
$\phi_{l}$ & $\mathrm{o}$ & Lower boundary of trial correction mass angular position \\
$\boldsymbol{M}$ & $\mathrm{kg}$ & Mass/Inertia matrix for rotor shaft and disks \\
$J$ & $\mathrm{~m}$ & Objective function \\
$N$ & & Population size \\
$N_{g}$ & & Maximum number of generation \\
$\boldsymbol{y}$ & $\mathrm{m}$ & Rotor displacement vector \\
$\omega_{\mathrm{o}}, \omega_{\mathrm{b}}$ & $\mathrm{rpm}$ & Rotor operation and balancing speeds \\
$\omega$ & $\mathrm{rad} \cdot \mathrm{s}^{-1}$ & Speed \\
$\boldsymbol{K}$ & $\mathrm{N} \cdot \mathrm{m}^{-1}$ & Stiffness matrix for shaft and bearings \\
$t$ & $\mathrm{~s}$ & Time \\
$m$ & $\mathrm{~kg}$ & Trial correction mass \\
$\emptyset$ & $\circ$ & Trial correction mass angular position \\
$\theta$ & $\mathrm{radian}$ & Unbalance angular position \\
$m_{\mathrm{u}}$ & $\mathrm{kg}$ & Unbalance mass \\
$\boldsymbol{f}_{\mathrm{ud}}$ & $\mathrm{N}$ & Unbalanced force \\
$m_{h}$ & $\mathrm{~kg}$ & Upper boundary of trial correction mass \\
$\phi_{h}$ & $\mathrm{o}$ & Upper boundary of trial correction mass angular position \\
$\boldsymbol{q}^{\mathrm{o}}$ & $\mathrm{N}$ & Vector of other force \\
$\boldsymbol{q}^{\mathrm{u}}$ & $\mathrm{N}$ & Vector of unbalanced force \\
$\dot{\boldsymbol{y}}$ & $\mathrm{m}$ & Velocity vector \\
\hline & & \\
\hline
\end{tabular}

Although the above techniques enable balancing procedures and minimize operating cost, they still have a few inherent shortcomings. For example, the techniques are non-universal, some approaches require two measurement instruments at one measurement point, and they take longer time and require high balancing speed as well as high professional training. In order to minimize these bottlenecks, the present work utilizes differential evolution (DE) and genetic algorithm (GA) as optimization techniques for obtaining correction parameters. The optimization methods consider gyroscopic effects, low balancing speed and measurements are taken in one direction only at each measuring point. The optimum correction parameters obtained do not only balance the rotor at balancing and operation speeds but also at higher speeds. Furthermore, the study presents the influence of correction parameters and compares the performance of the two optimization techniques.

\section{Formulation and implementation of unbalanced rotor problem}

Equation of motion of a rotor-bearing system with forced damped vibration can be assembled in general form as:

$$
M \ddot{y}+C \dot{y}+K y=q
$$

Then, for an unbalanced rotor-bearing system, Equation (1) can be written as:

$$
\boldsymbol{M} \ddot{\boldsymbol{y}}+\boldsymbol{C} \dot{\boldsymbol{y}}+\boldsymbol{K} \boldsymbol{y}=\boldsymbol{q}^{\mathrm{u}}+\boldsymbol{q}^{0}
$$

$\boldsymbol{f}_{\text {ud }}$ due to $m_{\mathrm{u}}$ located at node $j$ (make angle $\theta$ with respect to $Y$-axis) and at $t=0$ (when unbalanced rotor is at rest and position of $m_{\mathrm{u}}$ is as shown in Fig. 1) can be defined as:

$$
\left(\boldsymbol{f}_{\mathrm{ud}}\right)_{j}=m_{\mathrm{u}} r \omega^{2}\left[\begin{array}{c}
\sin (\omega t+\theta) \\
\cos (\omega t+\theta) \\
0 \\
0
\end{array}\right]
$$

Furthermore, equation (3) can be expressed as:

$$
\left\{\boldsymbol{q}^{\mathrm{d}}\right\}_{j}=m_{\mathrm{u}} r \omega^{2}\left[\begin{array}{c}
\sin (\omega t) \cos \theta \\
-\sin (\omega t) \sin \theta \\
0 \\
0
\end{array}\right]+m_{\mathrm{u}} r \omega^{2}\left[\begin{array}{c}
\cos (\omega t) \sin \theta \\
\cos (\omega t) \cos \theta \\
0 \\
0
\end{array}\right]
$$




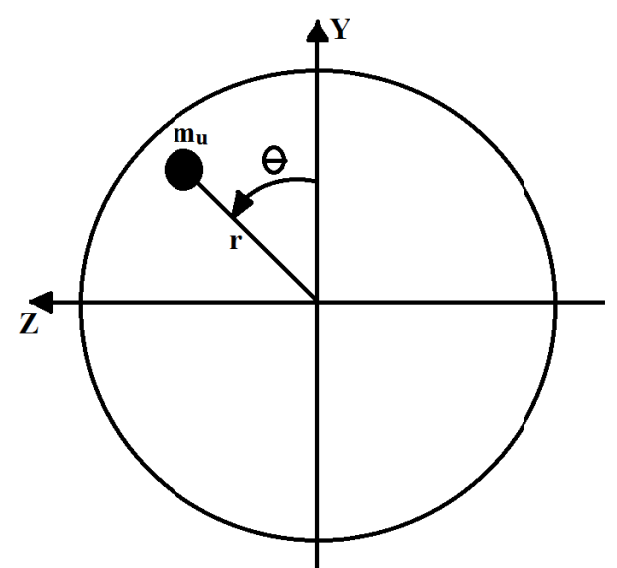

Fig. 1. Mass unbalance, $m_{\mathrm{u}}$ : unbalance mass, $r$ : distance from unbalance mass to shaft/rotor centerline, $\theta$ : angular position.

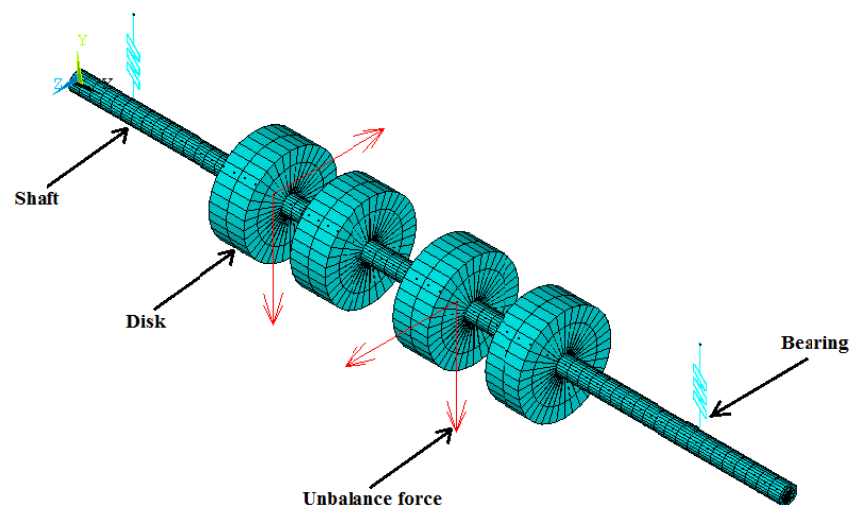

Fig. 2. Unbalance four-disk rotor model.

If the rotor bearing system contains unbalance, then $\boldsymbol{q}^{\mathrm{u}}=\boldsymbol{q}^{\mathrm{d}}$, else $\boldsymbol{q}^{\mathrm{u}}=0$. This work is focused at reducing the effect of $\boldsymbol{q}^{\mathrm{u}}$ on $\boldsymbol{y}$ using the two optimization techniques mentioned earlier. The procedures are implemented in ANSYS and MATLAB platforms.

An unbalanced rotor was modeled in ANSYS (Fig. 2) and the harmonic response analysis, based on equation (2), was conducted. More information on harmonic response analysis on ANSYS can be found in reference [10]. The rotor and its bearings were discretized using BEAM188 and COMBIN14 elements, respectively. BEAM188 is based on Timoshenko beam theory and it includes shear-deformation effects and can be used to analyze slender and stubby/thick beam structures. Each node has about six degrees of freedom which include three translations and three rotations. COMBIN14 has spring and damping capabilities and it is capable of longitudinal or torsional behaviour in 1,2 and 3D applications. CORIOLIS command was deployed to take care of the gyroscopic effect, SYNCHRO command was used to indicate that the force on the system is due to unbalance and stepped loading was utilized to determine how this unbalance force is being added to the system. In addition, ANSYS Parametric Design Language (APDL) script was used to perform both modal and harmonic analyses.

\section{Formulation of the optimization problem and evolutionary optimization techniques}

At each measurement point on the rotor-bearing system showing in Figure 2, a vector of amplitude of $\boldsymbol{y}$ will be obtained due to frequency steps. For $n$ measurement points and $j$ frequency steps, the $\boldsymbol{A} \boldsymbol{m}$ for each trial parameter in the $i$ th generation (where $i=1,2, \ldots, N_{\mathrm{g}}$ ) can be obtained as follows:

$$
A m=\left[\begin{array}{llll}
Y_{1} & Y_{2} & \ldots & Y_{\mathbf{n}}
\end{array}\right]_{j \times n}
$$

For each generation $i$ with $N$ solution, Equation (5) becomes:

$$
\boldsymbol{A m}=(\boldsymbol{A} \boldsymbol{m})_{T \times n}, T=N * j
$$

Therefore, the objective $J$ can be defined as:

$$
J=\operatorname{Minimize}(\max (\boldsymbol{A} \boldsymbol{m}))
$$

which is subject to:

$$
m_{l} \leqslant m \leqslant m_{h}, \emptyset_{l} \leqslant \emptyset \leqslant \emptyset_{h}
$$

In equation (7), the minimization will be done at any range of frequencies but the size of $\boldsymbol{A} \boldsymbol{m}$ for any $i$ th generation depends on the frequency steps employed and speed under consideration.

Various techniques based on evolutionary algorithm (EA) have been described and can be found in reference [11]. These include genetic algorithms (GA), genetic programming (GP), evolutionary programming (EP), differential evolution (DE), evolution strategies (ESs), cultural evolution (CE) and co-evolution (CoE). The well-known areas of applications include optimization of doublet [12], realistic process engineering optimization problems [13], Circuit Design Problems [14] among others. In this work, both DE and GA were used because they have been proven to be effective for evolutionary optimization. Interested reader is referred to the following references [11, 15-18].

\section{Proposed balancing methodology}

In DE the following processes are implemented: population generation, mutation, crossover, reproduction (selection) and elitism while those implemented for GA include: population generation, reproduction (selection), crossover, mutation and elitism in those orders. The flowchart for DE is shown in Figure 3.

Both techniques were deployed to minimize the maximum amplitudes (see Eq. (7)) at all measurement points in order to obtain optimal correction parameters. The number of correction parameters so obtained depends on the number of correction planes that are capable of reducing the unbalance to an acceptable level. Both ANSYS and MATLAB are used simultaneously and iteratively as summarized in Figure 4. The rotor modelling and analysis were done in ANSYS while MATLAB was used for 


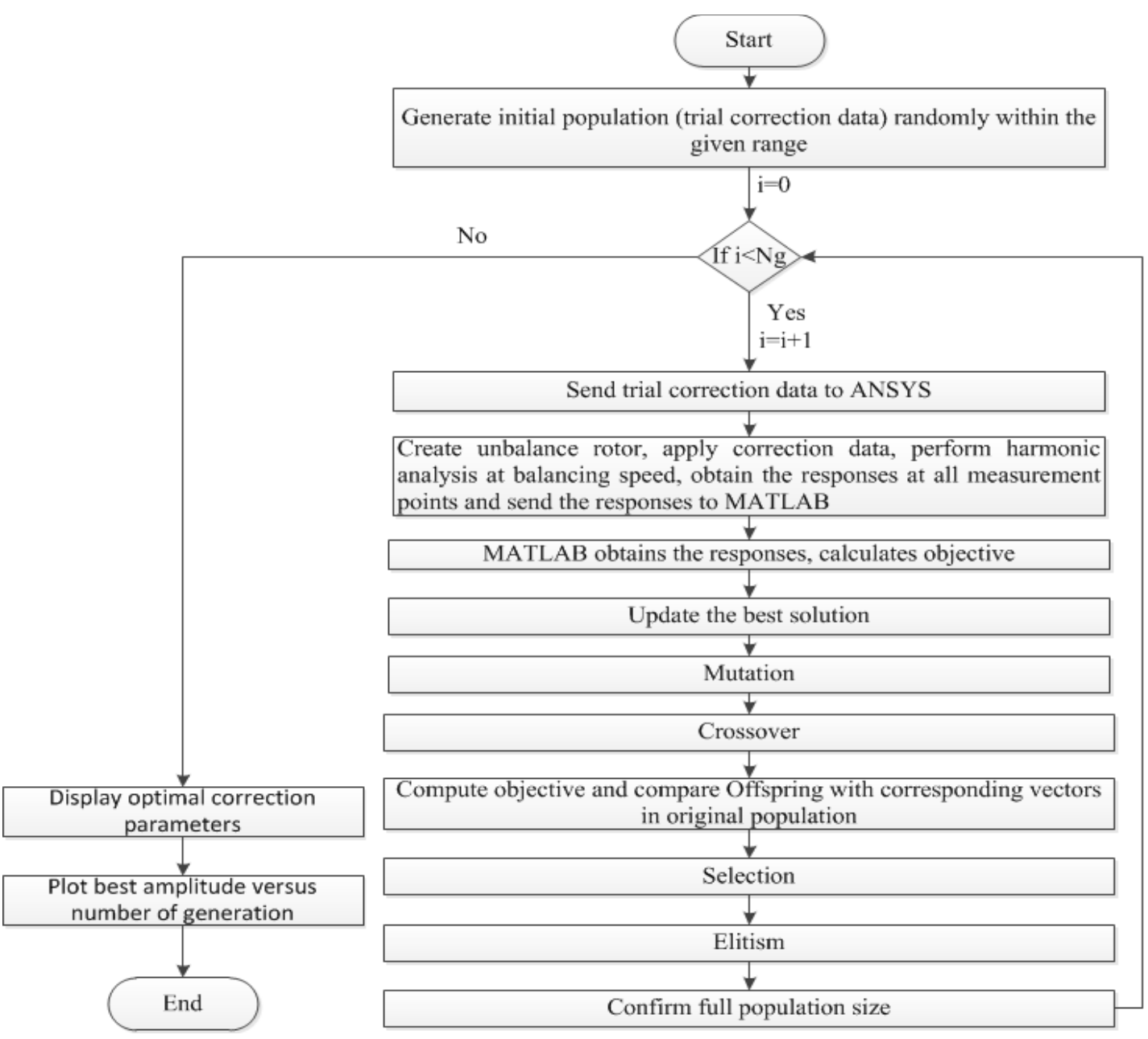

Fig. 3. Flowchart for balancing processes for DE.

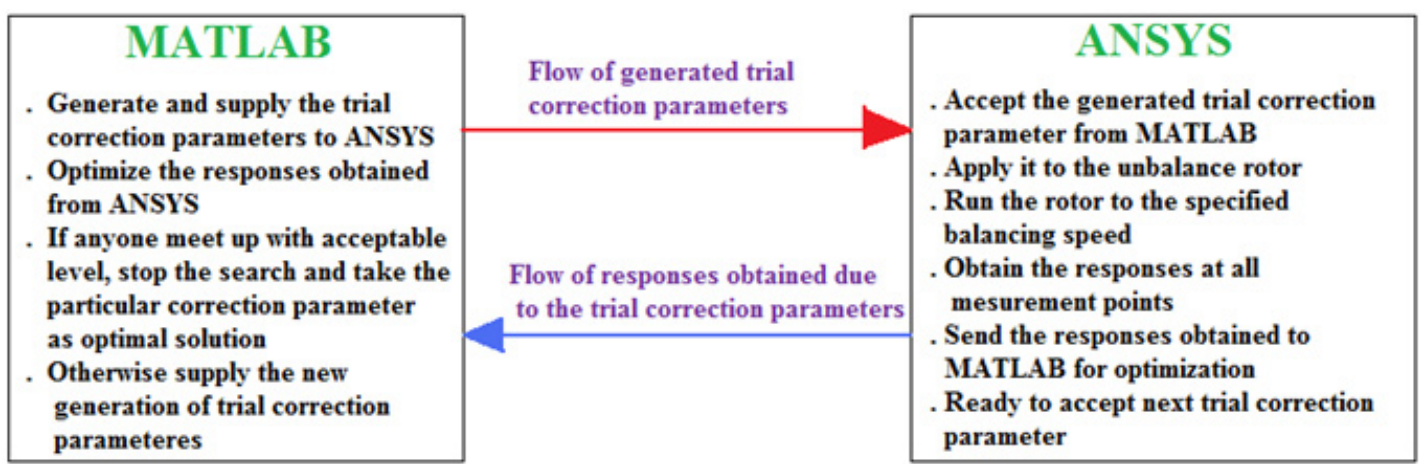

Fig. 4. The back-and-forth process for balancing methodology based on DE and GA.

the optimization process. For each iteration, the optimized correction parameters are generated in MATLAB and then transferred to ANSYS for the rotor analysis. The rotor responses obtained at measurement points in ANSYS are sent back to MATLAB for further processing (Fig. 4). This back-and-forth procedure continues until a stopping criterion (maximum number of generation or iteration) is met. Finally, the solution which gives the lowest value of the objective function is considered as the optimal correction parameters.

\section{Results and discussion}

To demonstrate the applicability of the proposed approaches, a four-disk rotor (Fig. 5) previously modelled in reference [6] was used for both DE and GA. The disk diameter, disk thickness, shaft diameter, density and elastic modulus are $0.08 \mathrm{~m}, 0.03 \mathrm{~m}, 0.015 \mathrm{~m}$, $7800 \mathrm{~kg} . \mathrm{m}^{-3}$ and $2.1 \times 10^{11} \mathrm{~N} . \mathrm{m}^{-2}$, respectively. Also, $K_{x x}$ and $K_{y y}$ are 140106 and 175133 N.m ${ }^{-1}$ while $C_{x x}$ and $C_{y y}$ are 12.26 and 17.51 N.s.m ${ }^{-1}$, respectively. In Figure 5, 
Table 1. Different cases of unbalance and the corresponding proposed correction parameters.

\begin{tabular}{|c|c|c|c|c|c|}
\hline Cases & $\begin{array}{c}\text { Unbalance } \\
\text { disks }\end{array}$ & $\begin{array}{l}\text { Unbalance with } \\
\text { location }\left(\mathrm{g} @^{\circ}\right)\end{array}$ & $\begin{array}{c}\text { Proposed } \\
\text { correction planes }\end{array}$ & $\begin{array}{l}\text { Correction } \\
\text { radii }(\mathrm{mm})\end{array}$ & $\begin{array}{l}\text { Measurement } \\
\text { points }\end{array}$ \\
\hline 1 & $\mathrm{~A}$ and $\mathrm{C}$ & $\begin{array}{l}U_{\mathrm{A}}=0.8 @ 100 \\
U_{\mathrm{C}}=1.2 @ 230\end{array}$ & $\begin{array}{c}19 \& 32,25 \& 32 \\
\text { and } 25 \& 38\end{array}$ & 36 & 1 and 2 \\
\hline 2 & $\mathrm{~A}$ and $\mathrm{C}$ & $\begin{array}{l}U_{\mathrm{A}}=0.8 @ 100 \\
U_{\mathrm{C}}=1.2 @ 230\end{array}$ & $\begin{array}{c}300 \& 302,301 \& \\
302 \text { and } 301 \& 303\end{array}$ & 40 & 1,2 and 3 \\
\hline 3 & $\mathrm{~A}, \mathrm{~B}, \mathrm{C}$ and $\mathrm{D}$ & $\begin{array}{l}U_{\mathrm{A}}=0.8 @ 100 \\
U_{\mathrm{B}}=2.0 @ 230 \\
U_{\mathrm{C}}=0.4 @ 0 \\
U_{\mathrm{D}}=0.5 @ 310\end{array}$ & $\begin{array}{c}19,25 \& 32 ; \\
25,32 \& 38 \text { and } \\
19,25 \& 38\end{array}$ & 36 & 1 and 2 \\
\hline 4 & $\mathrm{~A}, \mathrm{~B}, \mathrm{C}$ and $\mathrm{D}$ & $\begin{array}{l}U_{\mathrm{A}}=0.8 @ 100 \\
U_{\mathrm{B}}=2.0 @ 230 \\
U_{\mathrm{C}}=0.4 @ 0 \\
U_{\mathrm{D}}=0.5 @ 310\end{array}$ & $\begin{array}{c}300,301 \& 302 ; \\
301,302 \& 303 \\
\text { and } 300,301 \& \\
303\end{array}$ & 40 & 1,2 and 3 \\
\hline
\end{tabular}

Table 2. Comparison of the optimized correction parameters with those in reference [6].

\begin{tabular}{|c|c|c|c|c|c|c|c|c|c|}
\hline \multicolumn{9}{|c|}{ Present study } & 2 \\
\hline \multirow{2}{*}{$\begin{array}{c}\text { Correction } \\
\text { planes }\end{array}$} & \multicolumn{2}{|c|}{$\begin{array}{c}\text { Optimized } \\
\text { correction } \\
\text { parameters }\end{array}$} & & \multirow{2}{*}{$\begin{array}{c}\text { Correction } \\
\text { planes }\end{array}$} & \multicolumn{2}{|c|}{$\begin{array}{l}\text { Optimized } \\
\text { correction } \\
\text { parameters }\end{array}$} & \multirow{2}{*}{$\begin{array}{c}\text { Correction } \\
\text { planes }\end{array}$} & \multicolumn{2}{|c|}{$\begin{array}{l}\text { Optimized } \\
\text { correction } \\
\text { parameters }\end{array}$} \\
\hline & $\begin{array}{c}\text { Masses } \\
(\mathrm{g})\end{array}$ & $\begin{array}{l}\text { Angles } \\
\text { (Deg.) }\end{array}$ & & & $\begin{array}{c}\text { Masses } \\
(\mathrm{g})\end{array}$ & $\begin{array}{l}\text { Angles } \\
\text { (Deg.) }\end{array}$ & & $\begin{array}{c}\text { Masses } \\
\text { (g) }\end{array}$ & $\begin{array}{l}\text { Angles } \\
\text { (Deg.) }\end{array}$ \\
\hline \multirow{4}{*}{ Unknown } & \multirow{2}{*}{0.80} & \multirow{2}{*}{279.68} & \multirow{2}{*}{ DE } & 18 & 0.70 & 0.05 & 19 & 0.10 & 14.10 \\
\hline & & & & 31 & 0.32 & 38.88 & 32 & 0.80 & 11.58 \\
\hline & \multirow{2}{*}{0.19} & \multirow{2}{*}{50.29} & \multirow{2}{*}{ GA } & 18 & 0.30 & 70.61 & 19 & 0.92 & 15.65 \\
\hline & & & & 31 & 0.81 & 360.00 & 32 & 0.09 & 0.00 \\
\hline
\end{tabular}

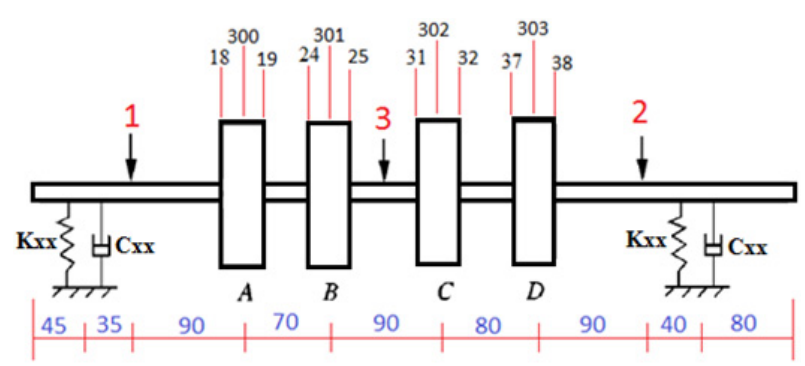

All dimensions are in $\mathrm{mm}$

Fig. 5. The four-disk rotor configuration considered for this work A-D: Rotor disks, 1, 2, 3: measuring points on the shaft, $18,24,31,37 ; 19,25,32,38$ and $300,301,302,303$ : nodes at the left, right and middle edges of the disks where the correction masses can be attached.

A, B, C, and D are rotor disks while 1,2 , and 3 denote measurement points in which point 3 is located at centre of the distance between disks $\mathrm{B}$ and $\mathrm{C}$. The proposed correction planes are identified as 300, 301, 302 and 303, and $18,19,24,25,31,32,37$ and 38 . However, it was assumed that $\omega_{\mathrm{o}}$ is $4000 \mathrm{rpm}$. The ANSYS model of a rotor with unbalances on two disks (A and C) is shown in Figure 2. The modal analysis of the rotor was initially performed and the first two critical speeds are 1874.7 and $4582.26 \mathrm{rpm}$. These critical speeds imply that $\omega_{\mathrm{o}}$ (which is $4000 \mathrm{rpm}$ ) is supercritical (i.e. above the first critical speed).

\subsection{Validation of DE and GA results}

Simulations were carried out based on 4 cases presented in Table 1 . In each case, the distance $r$ between the mass unbalance $m_{\mathrm{u}}$ and the rotor center (see Fig. 1) is $0.036 \mathrm{~m}$ while $\omega_{\mathrm{b}}, N$ and $N_{\mathrm{g}}$ are $2000 \mathrm{rpm}, 40 \mathrm{solu}-$ tions and 20 generations, respectively. Prior to this, the approach was compared with that of $\mathrm{Xu}$ et al. [6] by considering the case 1 as defined in Table 1 .

The ranges of $m$ and their $\phi$ were 0 to $0.003 \mathrm{~kg}$ and 0 to 360 degree, respectively. While reference [6] used 6 different $\omega_{\mathrm{b}}(1300,2100,2500,3000,4600,5600 \mathrm{rpm}), N$ of 80 and $N_{\mathrm{g}}$ of $20, \omega_{\mathrm{b}}$ of $2100 \mathrm{rpm}$ and the same $N$ and $N_{\mathrm{g}}$ were selected for the validation. Two trials, each having two correction planes (18 \& 31 and $19 \& 32$ ), were considered for balancing. Detailed results are presented in Table 2.

The first 2 critical speeds (1874.7 and $4582.26 \mathrm{rpm}$ ) are comparable to those obtained by reference [6] (i.e. 1871 and $5374 \mathrm{rpm}$ ). It is also observed that by considering the correction planes 18 and 31 for both DE and $\mathrm{GA}$, the correction masses are close to those of reference [6] while the correction angles are different. The correction planes that give similar results are opposite for the 2 approaches and the 2 cases considered. The slight difference between our results compared to those of reference [6] may be attributed to the facts that neither their exact locations of the unbalance on the 2 disks nor the correction planes were known. Also, the exact speed 

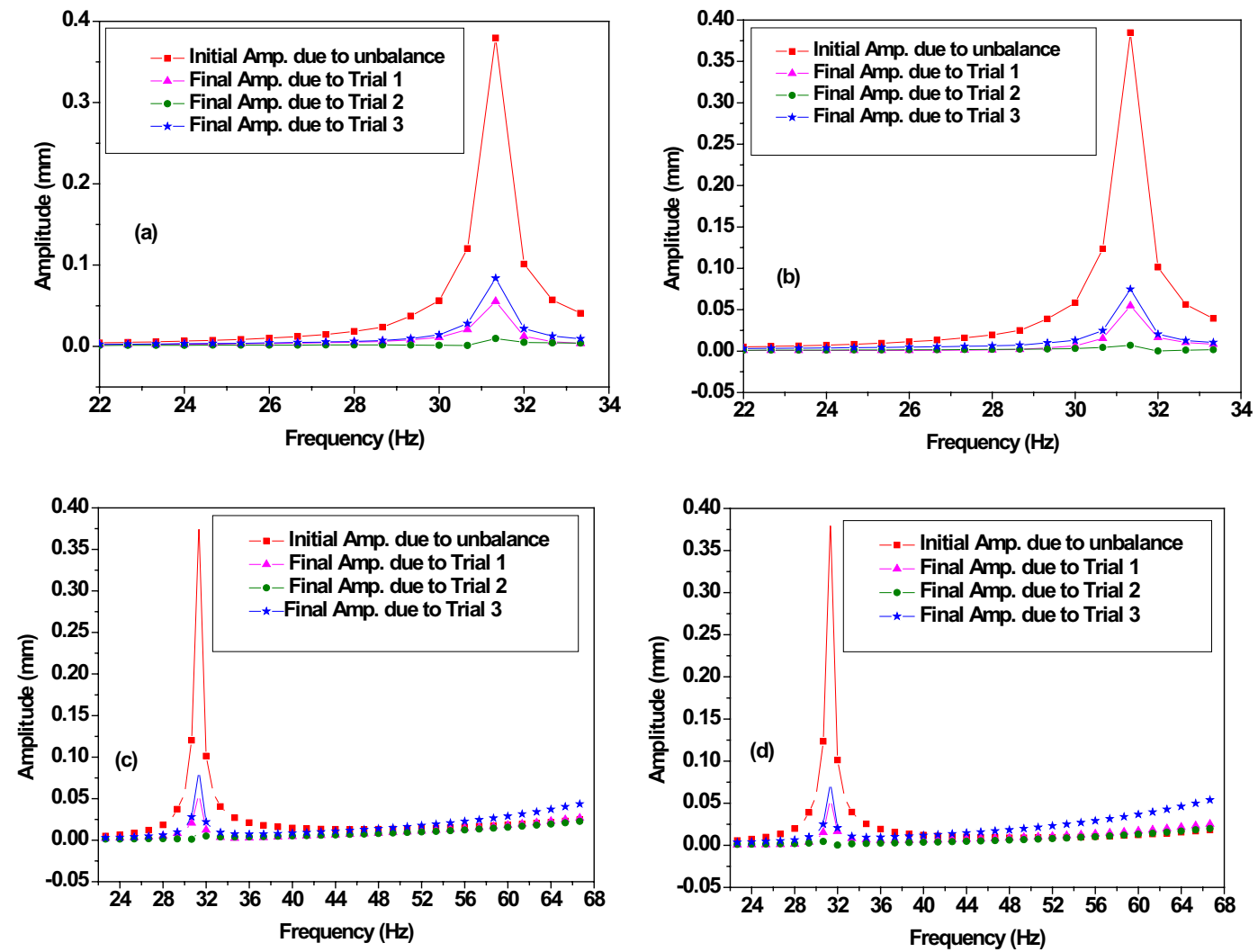

Fig. 6. Amp.: Amplitude, (a) comparison of the unbalanced and balanced rotors responses at $\omega_{\mathrm{b}}$ of 2000 rpm at first measurement point for Case 1 under DE, (b) comparison of the unbalanced and balanced rotors responses at $\omega_{\mathrm{b}}$ of $2000 \mathrm{rpm}$ at second measurement point for Case 1 under DE, (c) comparison of the unbalanced and balanced rotors responses at $\omega_{\mathrm{o}}$ of $4000 \mathrm{rpm}$ at first measurement point for Case 1 under DE, (d) comparison of the unbalanced and balanced rotors responses at $\omega_{\mathrm{o}}$ of $4000 \mathrm{rpm}$ at second measurement point for Case 1 under DE.

used for the simulation is not known. However, it was noted that $N_{\mathrm{g}}$ of 20 and $N$ of 80 took a long time to implement without a significant difference in the results, hence $N$ of 40 were considered for the rest of this work.

\subsection{Results of differential evolution (DE) based on cases 1 and 3}

To obtain the optimized correction parameters, DE was applied to all the cases listed in Table 1. While all the results are promising only the results of cases 1 and 3 are discussed here. In case 1 , disks $\mathrm{A}$ and $\mathrm{C}$ contain unbalance. Three trials were carried out and the optimized correction parameters obtained are presented in Table 3.

Each of the trial conditions in Table 3 was applied to the unbalanced rotor and tested at both $\omega_{\mathrm{b}}$ and $\omega_{\mathrm{o}}$. The responses are plotted against the initial response for the unbalanced rotor (Fig. 6). It is noted that the rotor is balanced at $\omega_{\mathrm{b}}$ with all the 3 correction parameters (Figs. $6 \mathrm{a}$ and $6 \mathrm{~b}$ ). However, only one out of these 3 correction parameters balanced the rotor at $\omega_{\mathrm{o}}$, while the other trial conditions failed prior to reaching $\omega_{\mathrm{o}}$ (Figs. 6c and 6d). The responses are the same for case 2 at $\omega_{\mathrm{b}}$ but 2 out of 3 correction parameters balanced the rotor at $\omega_{\mathrm{o}}$. This makes case 2 superior to case 1 .
Table 3. Optimized correction parameters for Case 1 based on DE.

\begin{tabular}{cccc}
\hline \multirow{2}{*}{ Trial S/N } & Correction planes & \multicolumn{2}{c}{$\begin{array}{c}\text { Optimized correction } \\
\text { parameters }\end{array}$} \\
\cline { 3 - 4 } & & $\begin{array}{c}\text { Masses } \\
(\mathrm{g})\end{array}$ & $\begin{array}{c}\text { Angles } \\
(\text { Deg. })\end{array}$ \\
\hline \multirow{2}{*}{1} & 19 & 0.4140 & 64.5597 \\
& 32 & 0.8040 & 0.0001 \\
2 & 25 & 0.3070 & 41.8438 \\
& 32 & 0.6780 & 0.0001 \\
3 & 25 & 0.9750 & 181.1602 \\
& 38 & 2.2680 & 12.8803 \\
\hline
\end{tabular}

In Figures 6-8, the response of unbalanced rotor is indicated as Initial Amp. due to unbalance while the responses of balanced rotor are designated as Final Amp. due to Trial 1, Trial 2 and Trial 3. The best of the correction masses from which balanced rotor responses were obtained are considered as desired correction masses.

In the case 3 , disks A, B, C and D contain unbalance and following the procedures described earlier, the optimization results are presented in Table 4.

In the same manner, each of the trials in Table 4 was applied to the unbalanced rotor and tested at 

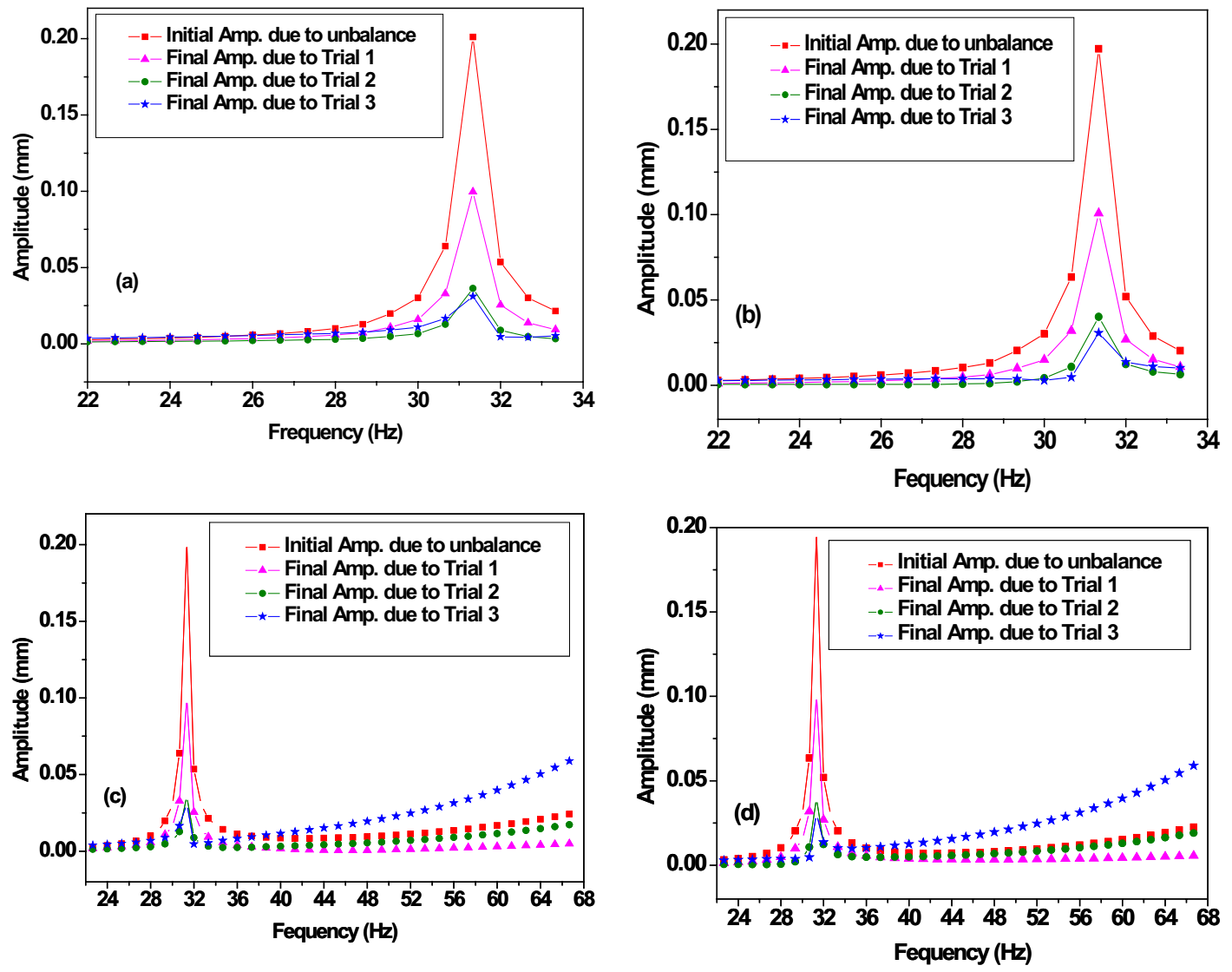

Fig. 7. Amp.: Amplitude, (a) comparison of the unbalanced and balanced rotors responses at $\omega_{b}$ of 2000 rpm at first measurement point for Case 3 under DE, (b) comparison of the unbalanced and balanced rotors responses at $\omega_{b}$ of $2000 \mathrm{rpm}$ at second measurement point for Case 3 under DE, (c) comparison of the unbalanced and balanced rotors responses at $\omega_{\mathrm{o}}$ of $4000 \mathrm{rpm}$ at first measurement point for Case 3 under DE, (d) comparison of the unbalanced and balanced rotors responses at $\omega_{\mathrm{o}}$ of $4000 \mathrm{rpm}$ at second measurement point for Case 3 under DE.

Table 4. Optimized correction parameters for Case 3 based on DE.

\begin{tabular}{cccc}
\hline \multirow{2}{*}{ Trial S/N } & Correction planes & \multicolumn{2}{c}{$\begin{array}{c}\text { Optimized correction } \\
\text { parameters }\end{array}$} \\
\cline { 3 - 4 } & & $\begin{array}{c}\text { Masses } \\
(\mathrm{g})\end{array}$ & $\begin{array}{c}\text { Angles } \\
\text { (Deg.) }\end{array}$ \\
\hline \multirow{2}{*}{1} & 19 & 1.7300 & 203.0158 \\
& 25 & 2.9000 & 0.0001 \\
& 32 & 2.1800 & 164.7053 \\
2 & 25 & 1.9400 & 217.5462 \\
& 32 & 2.0400 & 0.0001 \\
3 & 38 & 1.5200 & 136.3986 \\
& 19 & 2.5100 & 158.5715 \\
& 25 & 2.1600 & 297.3341 \\
& 38 & 1.3100 & 55.2829 \\
\hline
\end{tabular}

both $\omega_{\mathrm{b}}$ and $\omega_{\mathrm{o}}$. Based on the responses (Figs. 7a and 7b), all the correction parameters balanced the rotor at $\omega_{\mathrm{b}}$. However, Figures $7 \mathrm{c}$ and $7 \mathrm{~d}$ show that 2 of the 3 correction parameters balanced the rotor at $\omega_{\mathrm{o}}$. Similar responses were obtained at both $\omega_{\mathrm{b}}$ and $\omega_{\mathrm{o}}$ for the case 4 , although unbalances are reduced more in the case 3 compared to the case 4 .
Table 5. Optimized correction parameters for Case 1 based on GA.

\begin{tabular}{cccc}
\hline \multirow{2}{*}{ Trial S/N } & Correction planes & \multicolumn{2}{c}{$\begin{array}{c}\text { Optimized correction } \\
\text { parameters }\end{array}$} \\
\cline { 3 - 4 } & & $\begin{array}{c}\text { Masses } \\
(\mathrm{g})\end{array}$ & $\begin{array}{c}\text { Angles } \\
\text { (Deg.) }\end{array}$ \\
\hline \multirow{2}{*}{1} & 19 & 1.0450 & 11.2000 \\
& 32 & 0.0630 & 132.8600 \\
2 & 25 & 0.1090 & 112.6700 \\
& 32 & 0.9540 & 7.5900 \\
3 & 25 & 0.8620 & 7.7300 \\
& 38 & 0.1340 & 71.5500 \\
\hline
\end{tabular}

\subsection{Comparison between GA and DE}

For the GA optimization technique, the result of case 1 only is described here. Three trials were performed and the optimized correction parameters are presented in Table 5.

Each trial condition in Table 5 was applied to the unbalanced rotor and tested at both $\omega_{\mathrm{b}}$ and $\omega_{\mathrm{o}}$. By examining the responses (Fig. 8), all the correction parameters 

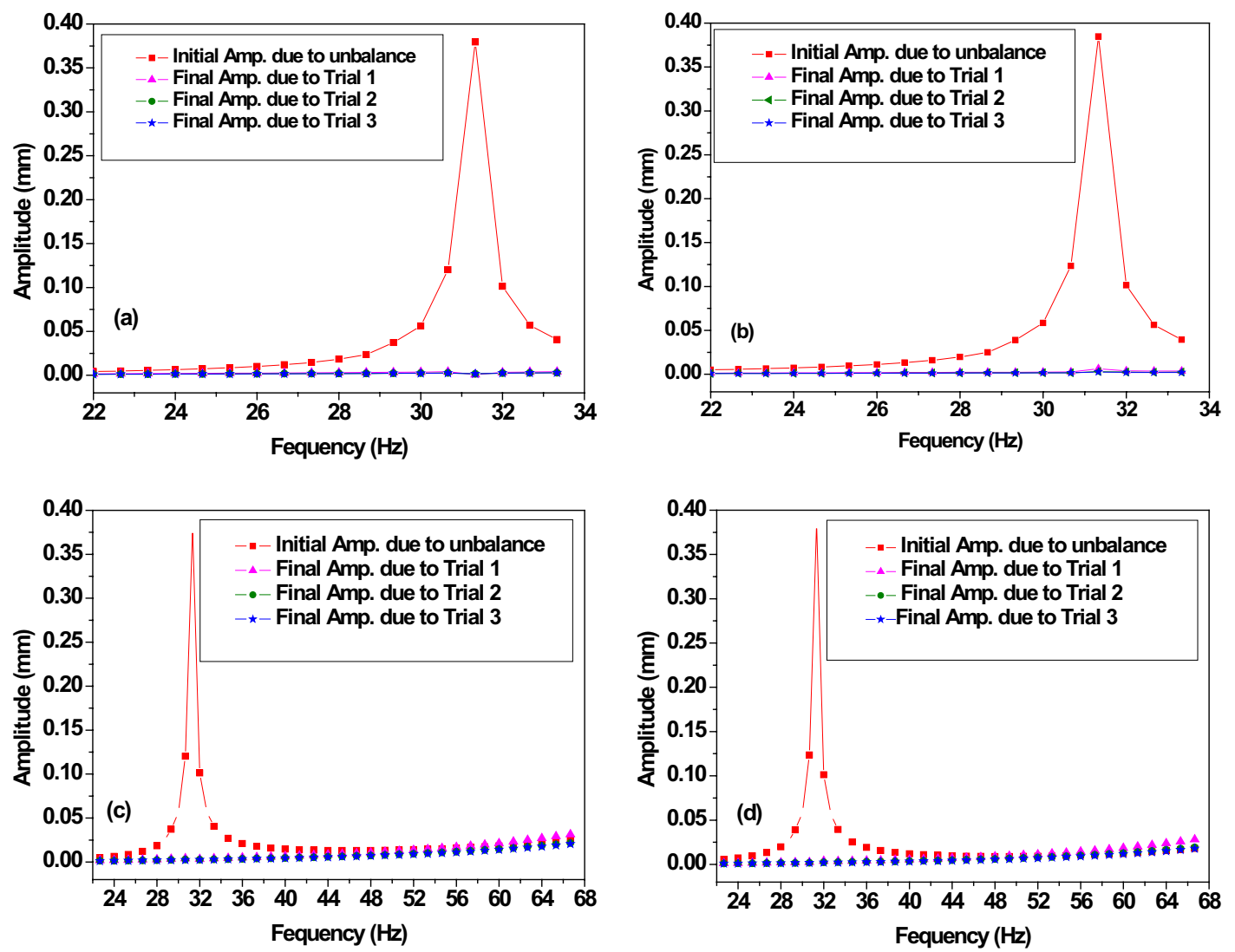

Fig. 8. Amp.: Amplitude, (a) comparison of the unbalanced and balanced rotors responses at $\omega_{b}$ of 2000 rpm at first measurement point for Case 1 under GA, (b) comparison of the unbalanced and balanced rotors responses at $\omega_{b}$ of $2000 \mathrm{rpm}$ at second measurement point for Case 1 under GA, (c) comparison of the unbalanced and balanced rotors responses at $\omega_{\mathrm{o}}$ of $4000 \mathrm{rpm}$ at first measurement point for Case 1 under GA, (d) comparison of the unbalanced and balanced rotors responses at $\omega_{\mathrm{o}}$ of $4000 \mathrm{rpm}$ at second measurement point for Case 1 under GA.

balanced the rotor at $\omega_{\mathrm{b}}$ (Figs. $8 \mathrm{a}$ and $8 \mathrm{~b}$ ) while 2 of the 3 conditions balanced the rotor at $\omega_{\mathrm{o}}$ (Figs. $8 \mathrm{c}$ and $8 \mathrm{~d}$ ).

As previously discussed, both DE and GA are excellent techniques for optimizing balancing parameters in unbalanced rotors. However, our results show that GA is more efficient than DE. One, the rotor responses (amplitudes of vibration) are much reduced in GA compared to that of DE (see Fig. 9 for the case 1). Two, optimization based on GA takes lesser time compared to DE. Three, the number of successful trials is more for GA than for DE.

\section{Conclusions}

Both DE and GA were successfully deployed as optimization techniques to balance an unbalanced flexible rotor. The balancing problem is formulated as an optimization problem with an objective of minimizing vibration amplitudes by applying different correction parameters. The optimal correction parameters so obtained keep the rotor balanced at both balancing speed and beyond. Our results also show that GA is slightly superior to DE. Also, unlike the available balancing techniques in the literature, the proposed method can be used to balance the

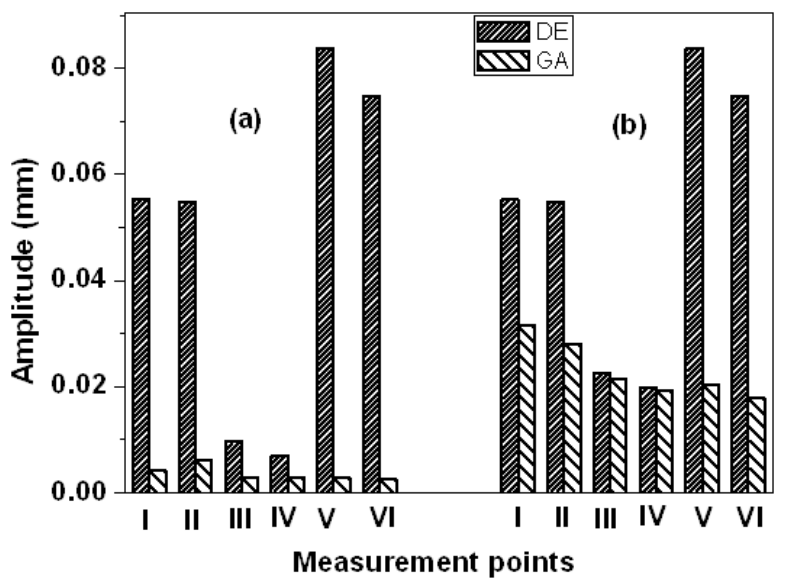

Fig. 9. Comparison between DE and GA at (a) $\omega_{\mathrm{b}}$ of $2000 \mathrm{rpm}$, (b) $\omega_{\mathrm{o}}$ of $4000 \mathrm{rpm}$ using Case 1.

rotor at low or high balancing speed, it requires only one measurement instrument at each measurement point, it saves cost and time, it does not require high professional training and the proposed approach follows neither modal nor influence-coefficient method approaches. However, 
experimental part validating the proposed technique will be carried out in the future work.

Acknowledgements. The authors acknowledge the support of King Fahd University of Petroleum \& Minerals (KFUPM), Saudi Arabia.

\section{References}

[1] M.J. Goodwin, Dynamics of Rotor-Bearing Systems, Unwin Hyman Ltd., London, 1989

[2] S. Liu, A modified low-speed balancing method for flexible rotors based on holospectrum, Mech. Syst. Signal Process. 21 (2007) 348-364

[3] S.G. Tan, X.X. Wang, A Theoretical Introduction to Low Speed Balancing of Flexible Rotors: Unification and Development of the Modal Balancing and Influence Coefficient Techniques, J. Sound Vib. 168 (1993) 385-394

[4] S. Liu, L. Qu, A new field balancing method of rotor systems based on holospectrum and genetic algorithm, Appl. Soft Comput. 8 (2008) 446-455

[5] W. Victor, Machinery Vibration: Balancing, McGrawHill, Inc., USA, 1995

[6] B. Xu, L. Qu, R. Sun, The Optimization TechniqueBased Balancing of Flexible Rotors without Test Runs, J. Sound Vib. 238 (2000) 877-892

[7] T. Yamamoto, Y. Ishida, Linear and Nonlinear Rotordynamics: A Modern Treatment with Applications, John Wiley \& Sons, Inc.. New York, 2001

[8] A.S. Das, M.C. Nighil, J.K. Dutt, H. Irretier, Vibration control and stability analysis of rotor-shaft system with electromagnetic exciters, Mech. Mach. Theory 43 (2008) $1295-1316$
[9] Y. Kang, T.-W. Lin, Y.-J. Chang, Y.-P. Chang, C.-C. Wang, Optimal balancing of flexible rotors by minimizing the condition number of influence coefficients, Mech. Mach. Theory 43 (2008) 891-908

[10] ANSYS, Ansys Structural Analysis Guide: ANSYS Release 10.0, in, USA, 2005

[11] A.P. Engelbrecht, Computational Intelligence An Introduction, 2nd edition, John Wiley \& Sons, Ltd, England, 2007

[12] D. Vasiljevic', J. Golobic', Comparison of the classical dumped least squares and genetic algorithm in the optimization of the doublet, First Online Workshop on Soft Computing (1996) 200-204

[13] T.T. Luong, Q.T. Pham, A Comparison Of The Performance Of Classical Methods and Genetic Algorithms For Optimization Problems Involving Numerical Models, IEEE (2003)

[14] A.M. Anile, V. Cutello, G. Nicosia, R. Rascuna', S. Spinella, Comparison among Evolutionary Algorithms and Classical Optimization Methods for Circuit Design Problems, IEEE (2005) 765-772

[15] R. Storn, K. Price, Differential Evolution - A Simple and Efficient Heuristic for Global Optimization over Continuous Spaces, Global Optimization 11 (1997) $341-359$

[16] A.A. Abou El Ela, M.A. Abido, S.R. Spea, Differential evolution algorithm for emission constrained economic power dispatch problem, Elect. Power Syst. Res. 80 (2010) 1286-1292

[17] M.A. Abido, Parameter optimization of multimachine power system stabilizers using genetic local search, Int. J. Electrical Power Energy Syst. 23 (2001) 785-794

[18] R. Chiong, O.K. Beng, A Comparison between Genetic Algorithms and Evolutionary Programming based on Cutting Stock Problem, Eng. Lett. 14 (2007) EL_14_1_14 